\title{
Characterizing the evolution of climate networks
}

\author{
L. Tupikina ${ }^{1,2}$, K. Rehfeld ${ }^{1,3}$, N. Molkenthin ${ }^{1,2}$, V. Stolbova ${ }^{1,2}$, N. Marwan ${ }^{1}$, and J. Kurths ${ }^{1,2}$ \\ ${ }^{1}$ PIK Potsdam Institute of Climate Impact Research, P.O. Box 601203, 14412 Potsdam, Germany \\ ${ }^{2}$ Department of Physics, Humboldt-Universität zu Berlin, Newtonstr. 15, 12489 Berlin, Germany \\ ${ }^{3}$ Alfred-Wegener-Institut, Helmholtz-Zentrum für Polar- und Meeresforschung, Telegrafenberg A43, 14473 Potsdam, \\ Germany \\ Correspondence to: L. Tupikina (tupikina@ pik-potsdam.de)
}

Received: 17 December 2013 - Revised: 27 March 2014 - Accepted: 5 May 2014 - Published: 25 June 2014

\begin{abstract}
Complex network theory has been successfully applied to understand the structural and functional topology of many dynamical systems from nature, society and technology. Many properties of these systems change over time, and, consequently, networks reconstructed from them will, too. However, although static and temporally changing networks have been studied extensively, methods to quantify their robustness as they evolve in time are lacking. In this paper we develop a theory to investigate how networks are changing within time based on the quantitative analysis of dissimilarities in the network structure.

Our main result is the common component evolution function (CCEF) which characterizes network development over time. To test our approach we apply it to several model systems, Erdős-Rényi networks, analytically derived flowbased networks, and transient simulations from the START model for which we control the change of single parameters over time. Then we construct annual climate networks from NCEP/NCAR reanalysis data for the Asian monsoon domain for the time period of 1970-2011 CE and use the CCEF to characterize the temporal evolution in this region. While this real-world CCEF displays a high degree of network persistence over large time lags, there are distinct time periods when common links break down. This phasing of these events coincides with years of strong El Niño/Southern Oscillation phenomena, confirming previous studies. The proposed method can be applied for any type of evolving network where the link but not the node set is changing, and may be particularly useful to characterize nonstationary evolving systems using complex networks.
\end{abstract}

\section{Introduction}

Networks are practical representations for complex systems with interacting components and have been used to study phenomena in sociology, engineering and natural systems (Barthélemy, 2011; Menck and Kurths, 2012; Palla et al., 2005; Holme et al., 2004). Complex network techniques, based on statistical associations between climate parameter time series at different points on Earth, have yielded new insights in the investigation of climate dynamics (Tsonis and Swanson, 2008; Donges et al., 2009; Paluš et al., 2011). Such climate networks have been used for detecting longrange correlations, or teleconnections (Martin et al., 2013; Barreiro et al., 2011), and studying such phenomena such as the El Niño/Southern Oscillation (ENSO, Gozolchiani et al., 2008; Deza et al., 2013) and the Indian Monsoon system (Rehfeld et al., 2013; Malik et al., 2011; Stolbova et al., 2014). In particular, Tsonis and Swanson (2008) found changes in the global network topology during El Niño events, with significantly fewer links and lower clustering coefficients, and inferred a lower predictability for El Niño over La Niña years. Using climate networks, Yamasaki et al. (2008) and Gozolchiani et al. (2008) also found ENSO influence on regional atmospheric processes in non-ENSO regions. Temporal and spatial variability of climate, and thus climate network structure, are of increasing interest considering ongoing environmental changes, and climate networks as evolving in time are still an open subject. The spatial-temporal developments in a given network set can be too complex to be captured by eye, and systematic approaches to quantify changes are needed. While Berezin et al. (2012) investigated the origins of the climate network stability such as the spatial 
embedding and physical coupling between climate in different locations using the correlation between correlation matrices, other studies describe how the network graph is changing over time to understand the behaviour of the underlying dynamical system (e.g. Rehfeld et al., 2013).

Various aspects of temporally changing networks have been considered for sociological and biological networks. Albert and Barabasi (2000) analysed random network growth and evolution in response to the addition or rewiring of links between nodes and found that the graph topology changed depending on the frequency of link changes. Fu et al. (2009) tracked node function changes using a stochastic block model for evolving networks to investigate evolutionary effects in email networks and gene regulation.

One of the most common dissimilarity measures which has been used for network comparison is Hamming distance. It was introduced by Hamming (1950) as a measure for comparing strings of symbols and was used for measuring the distance between the networks. Given the adjacency matrices $\mathbf{A}^{N}$ and $\mathbf{A}^{M}$ of two graphs $N$ and $M$, their Hamming distance is determined from the sum over the number of links which are found in one, but not the other network: $H(N, M)=$ $\sum_{i, j}\left|\mathbf{A}_{i, j}^{N}-\mathbf{A}_{i, j}^{M}\right|$. However, although Hamming distance can be generalized for directed networks with possibly differing node numbers, two networks $M$ and $N$ may have the same Hamming distance to the fixed network $K$ while having different topology themselves. The Hamming distance therefore may not enough to detect topological changes.

Here we propose a common component evolution function (CCEF) based on the common set of links in pairs of networks to evaluate graph changes quantitatively in space and time. We characterize the method using Erdós-Rényi networks (Erdös and Rényi, 1959), analytically derived flow networks (Molkenthin et al., 2014a, b) and transient simulations from the START model (Rehfeld et al., 2014) for which we control changes of individual parameters over time. Then we construct annual climate networks from NCEP/NCAR reanalysis data for the Asian monsoon domain and use the $\mathrm{CCEF}$ to characterize the temporal evolution in the monsoon system.

\section{Derivation of the common component evolution function}

We consider unweighted and undirected networks, for which $n$ nodes are joined in pairs by edges, or links. The linking structure is given in the adjacency matrix $\mathbf{A}$, a binary $n \times n$ matrix with zeros on the diagonal, as we do not allow for self-loops. An element is non-zero, $\mathbf{A}_{i j}=1$, if and only if the vertices $i$ and $j$ are connected, and zero otherwise.

Let us consider a linearly ordered set of $T$ evolving in time networks: $N_{1}, \ldots, N_{T}$. Then the common component network for two of these networks $N_{i}$ and $N_{j}, \mathrm{CC}\left(N_{i}, N_{j}\right)$, is a network on the same nodes, where the set of edges is present in both original networks. If $N_{i}$ and $N_{j}$ have adja- cency matrices $\mathbf{A}_{i}$ and $\mathbf{A}_{j}$, the number of edges in the common component network $\operatorname{CC}\left(N_{i}, N_{j}\right)$ is the number of nonzero elements above the diagonal in the binary sum of adjacency matrices $\mathbf{A}_{i}$ and $\mathbf{A}_{j}$. This common component network can be generalized for any $k+1$ networks by induction: $\mathrm{CC}\left(N_{i}, \ldots, N_{i+k+1}\right)=\mathrm{CC}\left(\mathrm{CC}\left(N_{i}, \ldots N_{i+k-1}\right), N_{i+k}\right)$.

The common component function $\operatorname{CCF}\left(N_{i}, \ldots, N_{i+k}\right)$ counts the number of links in a common component network of $k$ networks: $\operatorname{CCF}\left(N_{i}, \ldots, N_{i+k}\right)=$ $\left\|\mathrm{CC}\left(\mathrm{CC}\left(N_{i}, \ldots, N_{i+k-1}\right), N_{i+k}\right)\right\|$, where by $\left\|N_{i}\right\|$ we mean the number of links in the network $N_{i}$, and the common component function $\operatorname{CCF}\left(N_{i}, N_{k}\right)$ gives the number of coinciding edges in the graphs of $N_{i}$ and $N_{k}$, $i, k \in\{1,2, \ldots, T\}$. We set $\operatorname{CCF}\left(N_{i}\right)=\operatorname{CCF}\left(N_{i}, N_{i}\right)$. The common component function $\operatorname{CCF}\left(N_{i}\right)$ takes values in $\left[0, \max \operatorname{CCF}\left(N_{i}\right)\right]$ and is in the following normalized to $[0,1]$ using the maximal number of links in the networks.

In analogy to covariance estimation (Chatfield, 2004) and similar to Berezin et al. (2012), we take the mean over the CCFs with the same time lags to estimate the non-normalized common component evolution function, $\mathrm{CCEF}^{*}$, as

$\operatorname{CCEF}^{*}(\delta)=\frac{1}{T-\delta} \sum_{i=1}^{T-\delta} \operatorname{CCF}\left(N_{i}, N_{i+\delta}\right)$,

where $\delta$ is the time lag between the networks, and $\delta \in[0, T-$ 1]. The maximum value of the $\mathrm{CCEF}^{*}$ is given by $\operatorname{CCEF}^{*}(0)$ for zero lag, as an average number of links in the set of network

$\operatorname{CCEF}^{*}(0)=\frac{1}{T} \sum_{i=1}^{T} \operatorname{CCF}\left(N_{i}\right)$,

and we use it to obtain the normalized common component evolution function

$\operatorname{CCEF}(\delta)=\frac{\operatorname{CCEF}^{*}(\delta)}{\operatorname{CEEF}^{*}(0)}$,

which we will use exclusively in the following. As an estimation of the CCEF uncertainty we use the standard deviation over all CCEF values.

\subsection{Testing the method on random networks}

To test our method we generate a set of $T$ Erdős-Rényi graphs (Erdös and Rényi, 1959) with a fixed number of $n$ nodes and a fixed connection probability $p$. We artificially impose a linear ordering on the set, such that we can index them with $i \in(1, T)$. We compute the CCEF for ErdôsRényi graphs with 100 nodes and link probabilities of 0.3 , 0.5 , and 0.9. The resulting functions, shown in Fig. 1, decrease from 1 to a plateau at $\operatorname{CCEF}(\delta) \approx p$ for $\delta>0$ for each link probability $p$. For this example we can analytically compute the expected CCEF, since for $\delta=0$ each network is compared with itself and therefore $\mathrm{CCEF}=1$. For all other 


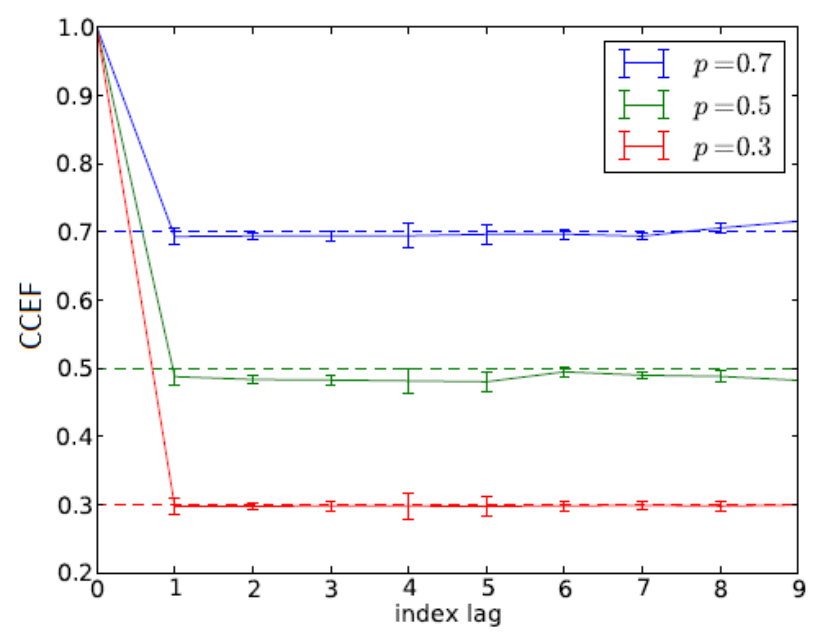

Figure 1. Mean CCEF for 10 indexed sets of random Erdôs-Rényi networks with 100 nodes and different linking probabilities $p$. The dashed lines correspond to the analytical CCEF levels, the error bars give the $1 \sigma$ standard deviation for each index lag

values of $\delta$ two random matrices with $n$ nodes and connection probability $p$ are compared. Then the number of totally possible links is $n(n-1) / 2$, and the expectation value of the number of links in each of the networks is $p n(n-1) / 2$. As the probability of each of the edges in one network to also appear in the other network is $p$, the total number of common links is $p^{2} n(n-1) / 2$, which with the normalization leads to $f(p)$, the ratio of total number of common links and the expectation value of the number of links to be

$f(p)=\frac{p^{2} n(n-1)}{p n(n-1)}=p$.

The CCEF for each linking probability therefore lies close to the expected value $p$.

\subsection{Test models}

To characterize our approach further we investigate simulations of more complex, spatially embedded processes. We obtained networks (i) analytically from flow fields, as described in Molkenthin et al. (2014a) and (ii) from the SpatioTemporally Autocorrelated Time series model START.

\subsubsection{Networks from flows}

The flow networks are constructed directly from a velocity field using a correlation measure based on the temperature profiles resulting from a temperature peak via advection and diffusion (Molkenthin et al., 2014b, a). The velocity function considered here is

$\boldsymbol{v}(x, y)=\left(\begin{array}{c}e^{\frac{-(y-0.5 x)^{2}}{c}} \\ 0.5 e^{\frac{-(y-0.5 x)^{2}}{c}}\end{array}\right)$, and we vary the parameter $c$ for the flow width from 200 to 2000 in 10 steps, thus gradually changing the flow network. The positions and the number of nodes are kept constant. For each value of $c$ we obtain a correlation matrices $\mathbf{C}_{1}, \ldots, \mathbf{C}_{10}$, and thresholding these matrices by different critical values we obtain set of adjacency matrices.

\subsubsection{Networks from the START model}

As a more complex test case we consider two transient simulations for the START model (Rehfeld et al., 2014). Networks generated from START undergo a distinct transition when the forcing parameter $F$ is changed: for $F=-1$ the network is partitioned into two vertical connected areas; for $F=0$ horizontal cross-links have appeared and link the two sections. At maximal forcing, for $F=1$, there is one large, horizontally oriented component. We performed two transient simulations with a $6 \times 7$ sampling grid (Rehfeld et al., 2014) for 20000 timesteps and 100 ensemble members each. In the first run the forcing parameter was increased linearly from the start to the end of the simulation. In the second run we periodically changed the forcing parameter $F(t)=\sin (t / 2000)$. Networks were constructed based on the $20 \%$ strongest links in the correlation matrices obtained for each 100-step-long time window. Due to the stochastic component, networks constructed for different ensemble members, but for the same time period, may be quite different, and networks for different periods of the same ensemble member may be quite similar.

\subsubsection{Asian monsoon data}

In a real-world application we used daily NCEP/NCAR reanalysis temperature anomaly data (NOAA, 2014) for the Asian monsoon domain for the years 1970-2010 CE. The spatial resolution was $2.5^{\circ} \times 2.5^{\circ}$, covering the area between $2.5^{\circ} \mathrm{S}-42.5^{\circ} \mathrm{N}$ and $57.5-122.5^{\circ} \mathrm{E}$, resulting in time series for 468 nodes. Networks were constructed using Pearson correlation in windows for each full year and by thresholding the correlation matrix such that we obtain a link density of $5 \%$. The same data set and time period was used in Molkenthin et al. (2014b) to investigate the influence of changing node topologies in space on the estimates of node degree and betweenness.

\section{Results}

\subsection{Flow networks}

We computed the CCEF for flow networks with linearly increasing flow-width parameter $c$. As Fig. 2 shows, the common component size decreases monotonically with the width parameter difference of the networks. The higher the threshold of the correlation matrix is, the faster the CCEF decays but the general shape does not change. 


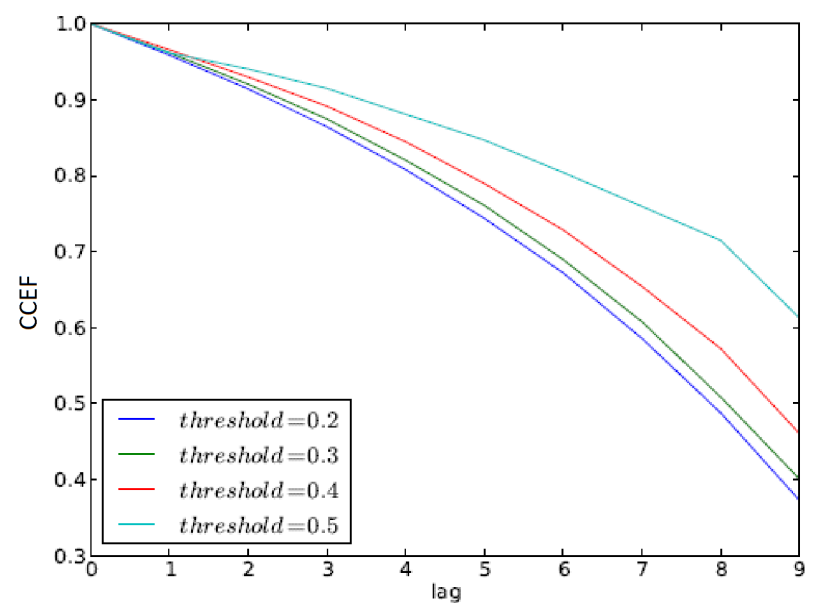

Figure 2. CCEF for the indexed flow networks with increasing flow-width parameter $c$, and for different threshold values of adjacency matrices of the flow networks.

\subsection{START-model networks}

The START model undergoes a more distinct transition from a network with two distinct parts through a connected stage with three regions to one single component (Rehfeld et al., 2014) in response to a single forcing parameter $F$. To characterize the CCEF response to different network evolution patterns we use two test cases, in which we vary $F$ from its minimum to its maximum. In the first example, the forcing parameter is varied linearly along time. The CCEF response is a slow decline from its maximum $\operatorname{CCEF}(0)=1$ to a minimum value $\operatorname{CCEF}(99) \approx 0.4$, as shown in Fig. 3 . In the second test the forcing parameter was varied periodically as a function of time, $\boldsymbol{F}=\sin \left(\frac{2 \pi}{P} \boldsymbol{t}\right)$, with $P=10$. In response to the sinusoidal forcing, periodic behaviour is also observable in the CCEF and with the same period as the forcing parameter.

\subsection{Application to the Asian Monsoon domain}

Finally, we used the CCEF to investigate the evolution of climate networks from observations. The networks were constructed using a link density of an annual basis for 41 years, 1970-2011 CE. The obtained CCEF in Fig. 4 is reminiscent of the Erdôs-Rényi networks in Sect. 2.1, with an initial quick decline followed by a plateau.

However, while in the case of Erdôs-Rényi networks (Fig. 1) the baseline is equal to the set link density, it is significantly higher than the link density here. We thus conclude that a high degree of persistence and a low amount of spatiotemporal variance can be found in climate networks from the Asian Monsoon domain at annual timescale.

The spatial domain selected for this study is the host of very distinct seasonal dynamics during the Asian Monsoon seasons, see e.g. Wang (2006). At inter-annual timescales,

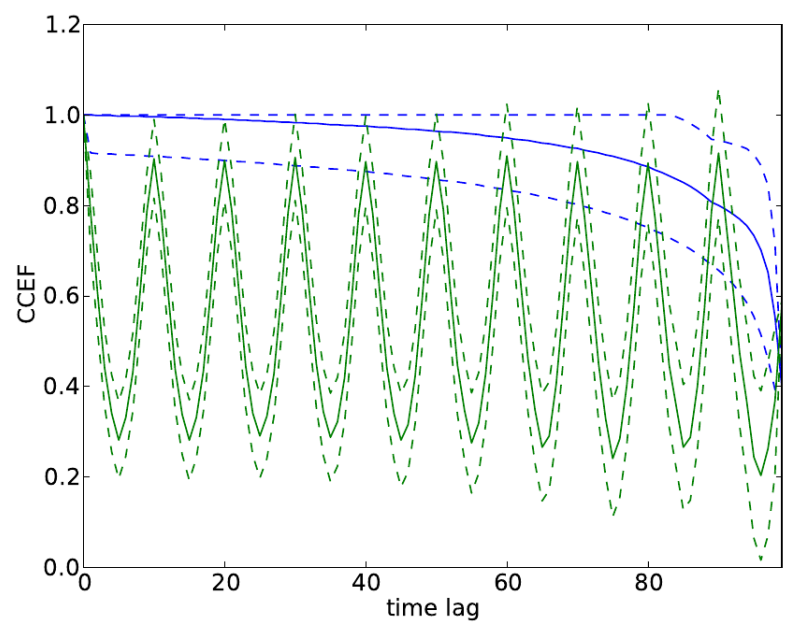

Figure 3. CCEF for networks from the START model with periodic (green) and linearly increasing (blue) forcing parameter $F$. The error bars indicate the standard deviation of the CC size estimates.

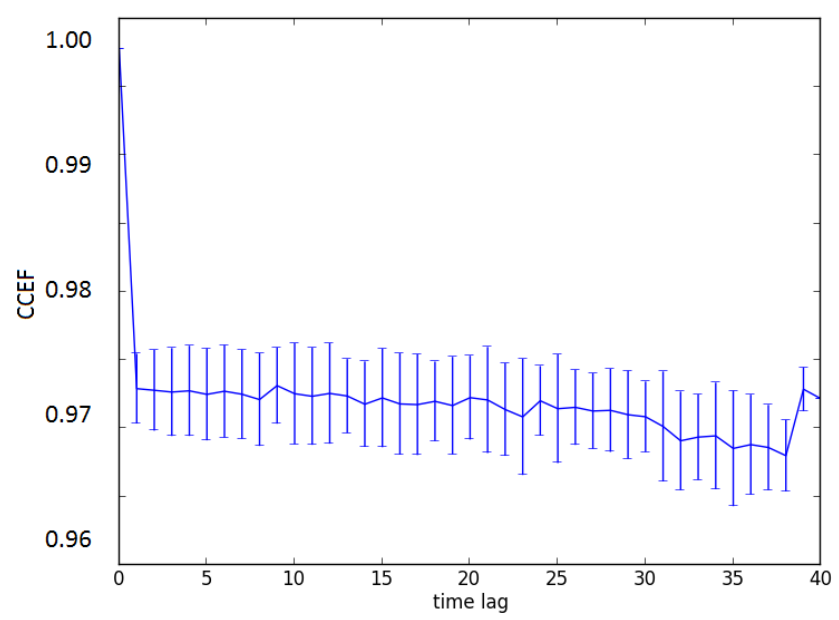

Figure 4. CCEF of annual climate networks for the time period 1970-2011 CE. Error bars are presented as CCEF standard deviation of the respective time lag in years.

however, teleconnections such as that to the ENSO phenomenon plays a significant role (Turner and Annamalai, 2012; Clarke, 2008). In order to identify reasons for the variability of climate networks in this region we compared the variation of common component functions $\operatorname{CCF}\left(N_{i}, N_{j}\right)$ for different $i, j \in[1970,2011]$, where $i$ is kept fixed. in this way, we obtained a common-links-recurrence diagram, illustrated in Fig. 5a, with maximum values on the diagonal. Each pair $(i, j)$ for $i, j \in[1970,2011]$ corresponds to the value of the common component function $\operatorname{CCF}\left(N_{i}, N_{j}\right)$, as in Eq. (3).

Figure 5a shows rows and columns with distinct lower values for the CCF. In these lines the overall sum, $S_{i}=$ $\sum_{j} \operatorname{CCF}\left(N_{i}, N_{j}\right)$, takes smaller values in the years $i \in$ (1971-1973, 1975, 1984, 1989, 1993, 1999). We compared this sequence with a list with strong El Niño phenomena 


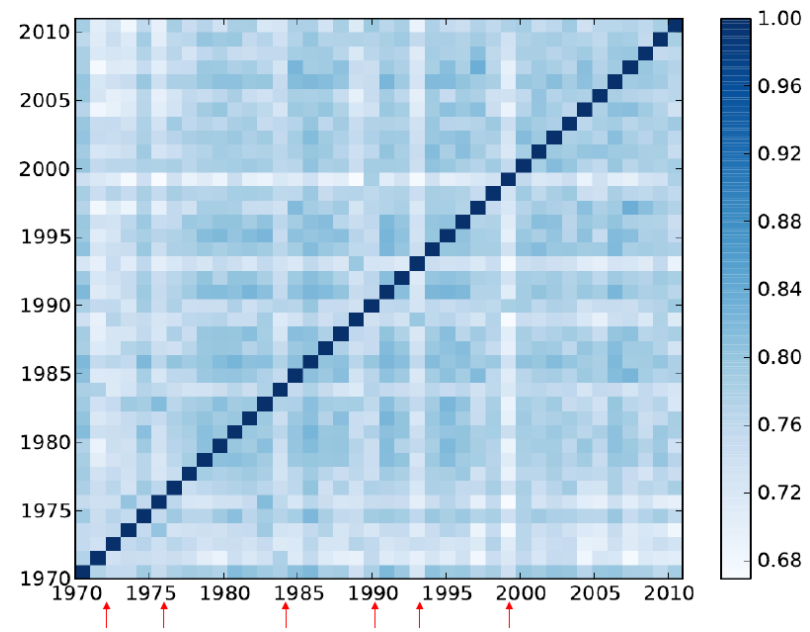

(a) Common link recurrence

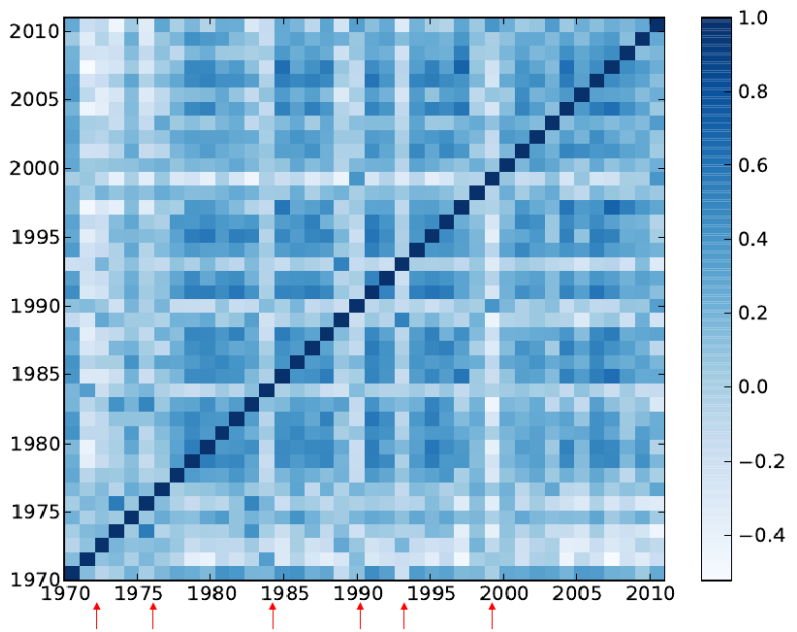

(b) Correlation of the common link evolution

Figure 5. Common-links-recurrence diagram (a) and correlation matrix of the common link evolution (b). Each point $(i, j)$ in diagram (b) corresponds to the value of the correlation coefficient $\operatorname{corr}(i, j)$ between the common component functions $\operatorname{CCF}\left(N_{i}, N_{k}\right)$ and $\operatorname{CCF}\left(N_{j}, N_{k}\right)$. Lines with low values (marked at the bottom with arrows) are observable around strong ENSO years.

according to the El Niño 3.4 index (Trenberth, 1997), and observed that 1972, 1982, 1988, 1992, and 1997 were the strongest ENSO event years in this time period. At the same time, the correlation between the CCF functions of these years and all others, given in Fig. 5b, also takes on very low values. Around stronger El Niño years the surface temperature networks have fewer common links, and the correlation of their CCF with all others is considerably lower. ENSO events occur during the Northern Hemisphere winter season and thus the main effect of the link breakdown in our networks occurs in the year after the event started.

\section{Discussion}

The CCEF enables us to investigate the evolution of linearly ordered, or evolving, network sets quantitatively. We tested its response to three different types of model networks and find that the responses enable us to characterize their evolution.

Unlike random networks, the flow-network CCEF level is, within limits, not related to the threshold value but displays a deterministic decrease of network similarity: two flow networks separated by bigger index lag have fewer links in the intersection, hence the common component function $\operatorname{CCF}\left(N_{i}, N_{i+\delta}\right)$ decreases with the growth of $\delta$, and the intersection of two flow networks decreases with the difference in the width parameter. Furthermore, we find that the links in a network set with higher threshold are more persistent.

The START model examples, on the other hand, illustrate the distinct difference between slow, linear changes of the processes generating the networks over time - and periodic, rapid transitions. While in the first case the CCEF decreases slowly, and only considerably for large time difference, in case of periodic and rapid transitions the CCEF response is also periodic over the time lag. In this case it is particularly important that the time window a single network corresponds to is sufficiently small compared to the ongoing evolution to avoid aliasing effects which would occur in the case of window width as a multiple of the forcing period and to be able to detect the changes at all.

The year-long daily temperature anomaly networks of the Asian Monsoon domain show a high degree of spatiotemporal persistence. This is consistent with the results of Berezin et al. (2012), who found similarly high values over large regions of the South Atlantic and the equatorial Pacific. While the general shape of the CCEF in Fig. 4 agrees with that for the Erdôs-Rényi random networks (Fig. 1), the CCEF fluctuates around a much higher level compared to the link density.

This points towards a highly non-random, deterministic general structure in the network on which the inter-annual variability is imprinted. Links in this network are comparatively stable but lose some of their stability when the external disturbance of an El Niño event is added. This agrees well with the findings of Gozolchiani et al. (2008) and Tsonis and Swanson (2008), who showed that, for global networks, fluctuations, or "blinking" of links could be related to the global signature of ENSO variability. Therefore, despite the large persistence in the monsoon network, the monsoon-ENSO teleconnection is also visible in the common link recurrence (Fig. 5b).

To check whether the main changes in the climate networks investigated here occurred due to changes in the degrees of "supernodes" (nodes with higher degree), we plotted the variability of the degree for each node in Fig. 6a) and find that, indeed, degree variability is high (low) where node degree is high (low). Computing the correlation between time 


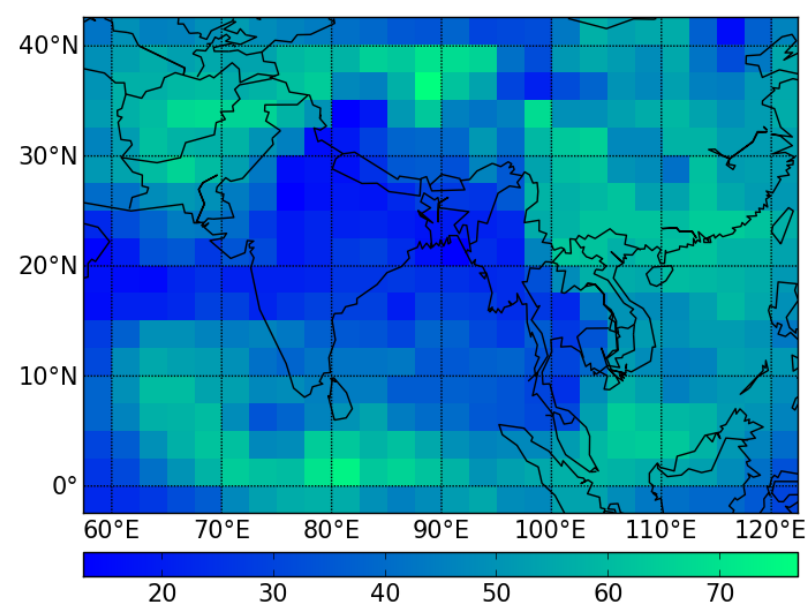

(a) Degree variability

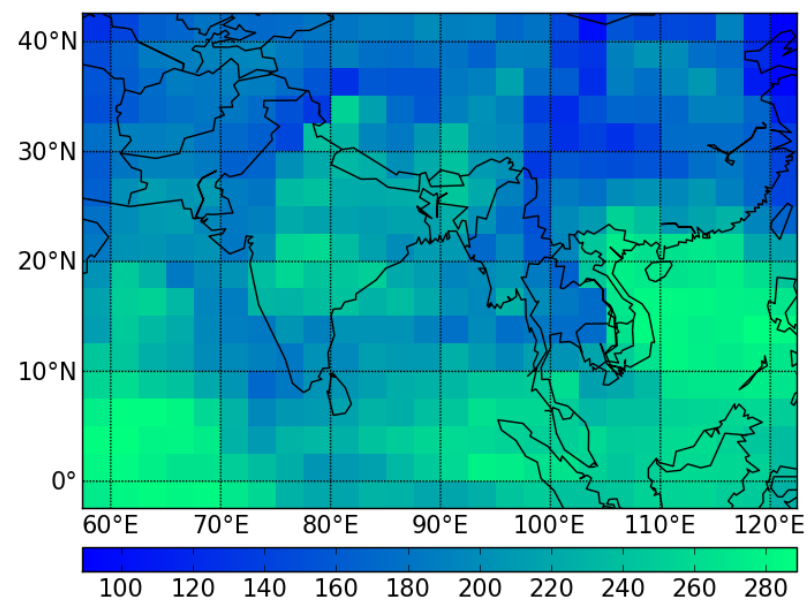

(b) Degree of the degree network

Figure 6. Mean degree variability (a) and degree of the degree variability (b) in the annual temperature anomaly networks (19702011).

series of node degrees, we obtain a network of degree variability. Figure $6 \mathrm{~b}$ shows the degree of the resulting graph. Where the degree variability is low, over mainland India, we find high degrees in the degree network, which means that links in this region are mostly persistent. Where the degree variability is high, over the adjacent Indian Ocean and the South China Sea, we also observe high degree values in the network of the degrees, suggesting that degree changes here are large, but synchronized. In the northern part of the Asian monsoon domain considered here, spanning from Afghanistan through Pakistan, the Himalayas to China, we find a higher degree variability with less synchronized degree changes. Desynchonization in this region may occur due to the additional effect of the continental Westerlies and the large altitudinal gradients.

\section{Conclusions}

We have presented a generic approach to characterize the evolution of networks. With model tests we established that it is possible to use it to distinguish random, deterministic and periodic evolution behaviours in a set of networks. The new quantity to measure variability and persistence in networks is suitable for different network types. For example, the network set may be linearly ordered by time - or by parameter difference. The method can be extended in a straightforward manner, but it currently requires that the node structure and link density remain constant. Applying the CCEF analysis to data from the Asian Monsoon domain we found that El Niño years are accompanied by a distinct network imprint, leading to small common components with non-ENSO years and high agreement with ENSO years. In the future, the CCEF could be a particularly useful tool in the investigation of change points in network evolution.

Acknowledgements. We thank Jakob Runge for helpful discussions. The authors acknowledge funding by the German Science Foundation (DFG Graduate School 1539), the German Federal Ministry for Education and Research (BMBF project PROGRESS) and the Marie-Curie ITN LINC (P7-PEOPLE-2011-ITN, grant no. 289447) and via Helmholtz grant VG-900NH.

Edited by: J. Davidsen

Reviewed by: two anonymous referees

\section{References}

Albert, R. and Barabasi, A.: Topology of evolving networks: local events and universality, Phys. Rev. Lett., 85, 5234-5237, 2000.

Barreiro, M., Marti, A., and Masoller, C.: Inferring long memory processes in the climate network via ordinal pattern analysis, Chaos, 21, 013101, doi:10.1063/1.3545273, 2011.

Barthélemy, M.: Spatial networks, Phys. Rep., 499, 1-100, doi:10.1016/j.physrep.2010.11.002, 2011.

Berezin, Y., Gozolchiani, A., Guez, O., and Havlin, S.: Stability of climate networks with time, Scientific Reports, 2, 666, doi:10.1038/srep00666, 2012.

Chatfield, C.: The analysis of time series: an introduction, CRC Press, Florida, USA, 6th Edn., 2004.

Clarke, A.: An introduction to the dynamics of El Niño \& the Southern Oscillation, Academic Press, 2008.

Deza, J., Barreiro, M., and Masoller, C.: Inferring interdependencies in climate networks constructed at inter-annual, intra-season and longer time scales, Eur. Phys. J.-Spec. Top., 222, 511-523, doi:10.1140/epjst/e2013-01856-5, 2013.

Donges, J., Zou, Y., Marwan, N., and Kurths, J.: Complex networks in the climate system, Eur. Phys. J.-Spec. Top., 174, 157-179, doi:10.1140/epjst/e2009-01098-2, 2009.

Erdös, P. and Rényi, A.: On random graphs I., Publicationes Mathematicae (Debrecen), 6, 290-297, 1959.

Fu, W., Song, L., and Xing, E. P.: Dynamic mixed membership blockmodel for evolving networks, in: Proceedings of the 26th 
Annual International Conference on Machine Learning - ICML '09, 1-8, doi:10.1145/1553374.1553416, 2009.

Gozolchiani, A., Yamasaki, K., Gazit, O., and Havlin, S.: Pattern of climate network blinking links follows El Niño events, EPL, 83, 28005, doi:10.1209/0295-5075/83/28005, 2008.

Hamming, R. W.: Error detecting and error correcting codes, The Bell System Technical Journal, 29, 147-160, doi:10.1002/j.1538-7305.1950.tb00463.x, 1950.

Holme, P., Edling, C. R., and Liljeros, F.: Structure and time evolution of an Internet dating community, Soc. Networks, 26, 155174, doi:10.1016/j.socnet.2004.01.007, 2004.

Malik, N., Bookhagen, B., Marwan, N., and Kurths, J.: Analysis of spatial and temporal extreme monsoonal rainfall over South Asia using complex networks, Clim. Dynam., 39, 971987, doi:10.1007/s00382-011-1156-4, 2011.

Martin, E. A., Paczuski, M., and Davidsen, J.: Interpretation of link fluctuations in climate networks during El Niño periods, EPLEurophys. Lett., 102, 48003, doi:10.1209/0295-5075/102/48003, 2013.

Menck, P. and Kurths, J.: Topological Identification of Weak Points in Power Grids, Nonlinear Dynamics of Electronic Systems, 144-147, 2012.

Molkenthin, N., Rehfeld, K., Marwan, N., and Kurths, J.: Networks from Flows - From Dynamics to Topology, Scientific Reports, 4, 4119, doi:10.1038/srep04119, 2014a.

Molkenthin, N., Rehfeld, K., Stolbova, V., Tupikina, L., and Kurths, J.: On the influence of spatial sampling on climate networks, Nonlin. Processes Geophys., 21, 651-657, doi:10.5194/npg-21651-2014, 2014b.

NOAA: NCEP/NCAR, http://www.esrl.noaa.gov/psd/data/gridded/ data.ncep.reanalysis.html (last access: February 2014), 2014.

Palla, G., Derényi, I., Farkas, I., and Vicsek, T.: Uncovering the overlapping community structure of complex networks in nature and society, Nature, 435, 814-818, doi:10.1038/nature03607, 2005.
Paluš, M., Hartman, D., Hlinka, J., and Vejmelka, M.: Discerning connectivity from dynamics in climate networks, Nonlin. Processes Geophys., 18, 751-763, doi:10.5194/npg-18-751-2011, 2011.

Rehfeld, K., Marwan, N., Breitenbach, S. F. M., and Kurths, J.: Late Holocene Asian Summer Monsoon dynamics from small but complex networks of palaeoclimate data, Clim. Dynam., 41, 3-19, doi:10.1007/s00382-012-1448-3, 2013.

Rehfeld, K., Molkenthin, N., and Kurths, J.: Testing the detectability of spatio-temporal climate transitions from paleoclimate networks with the START model, Nonlin. Processes Geophys., 21, 691-703, doi:10.5194/npg-21-691-2014, 2014.

Stolbova, V., Martin, P., Bookhagen, B., Marwan, N., and Kurths, J.: Topology and seasonal evolution of the network of extreme precipitation over the Indian subcontinent and Sri Lanka, Nonlin. Processes Geophys., in review, 2014.

Trenberth, K. E.: The Definition of El Niño, B. Am. Meteorol. Soc., 78, 2771-2777, 1997

Tsonis, A. and Swanson, K. L.: Topology and Predictability of El Niño and La Niña Networks, Phys. Rev. Lett., 100, 228502, doi:10.1103/PhysRevLett.100.228502, 2008.

Turner, A. and Annamalai, H.: Climate change and the South Asian summer monsoon, Nature Climate Change, 2, 587-595, doi:10.1038/nclimate1495, 2012.

Wang, B.: The Asian Monsoon, Springer-Verlag Berlin Heidelberg New York, ISBN 3-540-40610-7, 2006

Yamasaki, K., Gozolchiani, A., and Havlin, S.: Climate Networks around the Globe are Significantly Affected by El Niño, Phys. Rev. Lett., 100, 228501, doi:10.1103/PhysRevLett.100.228501, 2008. 